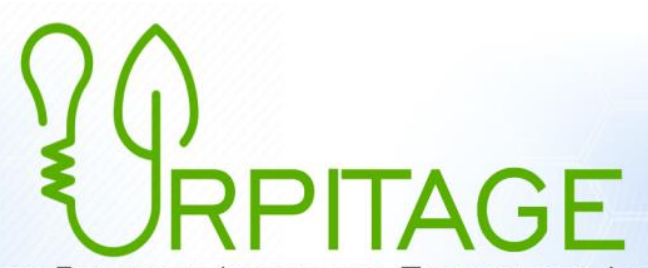

INTERNATIONAL REVIEW of PRACTICAL INNOVATION, TECHNOLOGY AND GREEN ENERgY

\title{
Survey of Leading Commodities Of Aceh Province As Academic Effort To Join And Build The Country
}

\author{
Mohd.Heikal, Muammar Khaddafi, Falahuddin, Ichsan, Fuadi, Dammanhur, Rico \\ Nur Ilham, Ahmad Fauzul Hakim Hasibuan, Munandar, Chalirafi
}

Faculty of Economics and Business, Universitas Malikussaleh

E-mail: riconurilham@unimal.ac.id

\begin{abstract}
Local governments must be able to prioritize superior commodities which are considered as the basis for sub-sectors to optimize regional economic advantages. Understanding the priorities of development planning and commodities in the agricultural, plantation and livestock sub-sectors in Aceh Province, it is necessary to know which agricultural, plantation and livestock sub-sectors are the leading and leading commodities in the agricultural, plantation and livestock sectors in Aceh Province. This research for community service was carried out on September 25, 2020 - August 25, 2021 with the location survey method. The criteria used based on this survey are 7 leading commodities in Aceh Province which include red chilies, cayenne peppers, shallots, beef, chicken meat, rice and eggs. The results of this service show that the agriculture and plantation sub-sectors are superior commodities from the period September 2020 - August 2021 with 9 regional locations in Aceh Province. Likewise with the nominated livestock sub-sector as a superior commodity in Aceh Province.
\end{abstract}

Keywords:Agriculture, Plantation, Livestock, Superior,Community Empowerment, Service

\section{INTRODUCTION}

National development is the welfare of the community by always seeking to improve the standard of living. In Law Number 32 of 2004 it is explained that regional development plays a very important role in national development. The Central Government has provided opportunities for local governments to regulate and manage their own regions in accordance with the principles of Regional Autonomy and Co-Administration. The principles of regional autonomy and assistance tasks are directed at accelerating the realization of community welfare through improvement, service, empowerment and participation of the community and academics in order to increase regional competitiveness by taking into account the principles of democracy. Based on the Regulation of the Minister of Home Affairs Number 9 of 2014 concerning Guidelines for the Development of Regional Superior Products,

Local government in implementing regional autonomy based on efficiency principles and effectiveness by taking into account the aspects regional potential and diversity regions in the face of competitive challenges global. Indonesia is a country with dominance of rural areas. The dominance of rural areas in Indonesia is not only shown by the large area of rural areas, but also by the large number of residents in rural areas. Rural areas have landscapes with the dominance of agricultural spatial patterns by relying on livelihoods from existing natural resources. Most of the rural areas are still an agrarian pattern, which is characterized by the livelihoods of the people who work in agriculture, which consists of food farming, fisheries, animal husbandry, plantations and 


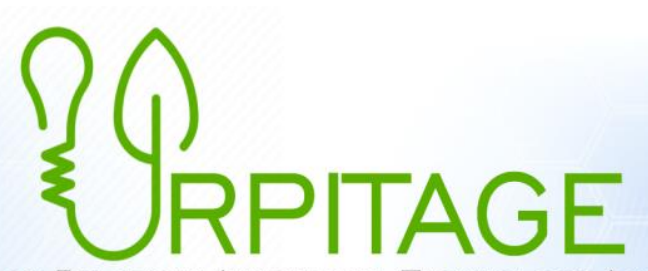

INTERnational REVIEW of Practical InNOVAtion, Technology AND GREen ENERgy

forestry. In addition, the current economic challenges in Indonesia, based on the 2020 literacy index, are only on a "medium" to "good" scale, which is 3.47 out of 5.00 .

The Producer Price Survey Report was prepared in order to support Bank Indonesia (BI) in updating the data on the Strategic Food Price Information Center (PIHPS), which is an information center containing the prices of 10 strategic food commodities with the aim of monitoring prices, coordinating policies and facilities to expand access to information for consumers. public. Aceh Province is one of the provinces that has long developed the agricultural sector which includes food crop agriculture, plantations, livestock and fisheries as the most superior commodities. Aceh Province in developing superior commodities as one of the country's development capital cannot be separated from various regional development problems, both general in nature and those of a regional strategy, such as; poverty and underdeveloped areas. Therefore,

\section{METHOD}

The survey was carried out by going directly to the field by surveying 7 dominant main commodities, namely red chili, cayenne pepper, red onion, beef, chicken meat, rice and eggs along with the quality of the commodities. The area surveyed is the working area of the Lhokseumawe Bank Indonesia Representative Office which consists of 10 Regencies/Cities in Aceh Province. This survey was participated by 25 participants from various regions in Aceh Province from September 25, 2020 to August 25, 2021. This survey presented 2 resource persons, namely the Head of Bank Indonesia Lhokseumawe City, Head of the Human Resource Management Doctoral Program at Batam University and 25 farmers with superior commodities in Aceh Province.

The preparation of service activities includes deliberations with the Head of Bank Indonesia, Lhokseumawe City and the Head of the Doctoral Program in Human Resource Management, University of Batam for the implementation of service activities. The preparation of this survey was coordinated directly with the plan to develop a country related to the Aceh Province's leading commodities as an effort of participating academics. For this survey, structured activities were designed.

\section{RESULTS}

The problems faced by society today began with the development of technology 4.0 in Indonesia since 2019, namely (1) causing an increase in global competitiveness through cooperation and confederation of companies. It can be seen that products will no longer be made by a worker in the future, but by robots or programmers and it doesn't take a long time to process a product, so that it can make it easier for companies to accelerate product marketing, and for marketing, this change should be able to carry out a strategy positioning. Positioning strategy is a very important factor in increasing the strength of the company's market position. In addition, a positioning strategy is needed as a step in determining market segments for businesses, (2) In addition to the impact of the Industrial revolution 4. 0 is one of the main drivers of increasing company revenues, overcoming financial difficulties in business, and can increase the country's GDP growth and (3) The impact of the 4.0 Industrial revolution is to provide a platform for further innovation bases with developing technologies. Manufacturing systems and services can be further developed. For example, with the application of accounting bookkeeping, the more development of accounting bookkeeping technology, the more it can make it easier for business people to clean up their books, one bookkeeping that can make it easier for you is to use Harmony Accounting Services. 0 is to provide a platform for further innovation base with developing technology. Manufacturing systems and services can be further developed. For example, with the application of accounting bookkeeping, the 


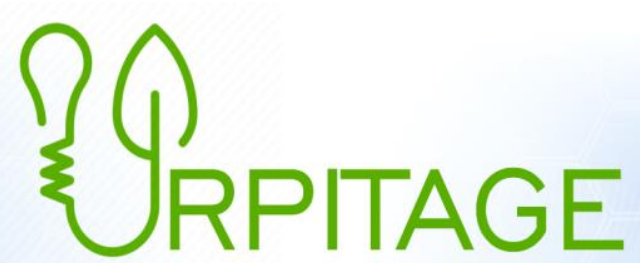

INTERNATIONAL REVIEW OF PRACTICAL INNOVATION, TECHNOLOGY AND GREEN ENERGY

more development of accounting bookkeeping technology, the more it can make it easier for business people to clean up their books, one bookkeeping that can make it easier for you is to use Harmony Accounting Services. 0 is to provide a platform for further innovation base with developing technology. Manufacturing systems and services can be further developed. For example, with the application of accounting bookkeeping, the more development of accounting bookkeeping technology, the more it can make it easier for business people to clean up their books, one bookkeeping that can make it easier for you is to use Harmony Accounting Services.

Table 1. Survey of Leading Commodity Producers of Aceh Province

\begin{tabular}{|c|c|c|c|c|c|c|c|c|c|c|c|c|c|c|c|}
\hline № & Tahun & Bulan & $\mathrm{Tg} 1$ & Kota/Kab & Komoditas & Kualitas & Produsen & Alamat & KTR Res & Hр & Harga & merek & $\begin{array}{c}\text { umur } \\
\text { tanaman/ternak }\end{array}$ & $\begin{array}{l}\text { Mulai tanam/ } \\
\text { mulai ternak }\end{array}$ & Keterangan \\
\hline 1 & 2021 & Agustus & 19 & Aceh Timur & Cabe merah & Keriting Segar & llyas & Desa Blang Gleum & Via phone & 0823-9219-3065 & 20.000 & & & & 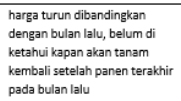 \\
\hline 2 & 2021 & Agustus & 19 & Aceh Timur & Cabe Rawit & Hijau Segar & Ilyas & Dess Blang Gleum & \begin{tabular}{|l|} 
Via phone \\
\end{tabular} & \begin{tabular}{|l|} 
0823-9219-3066 \\
\end{tabular} & 25.000 & & 205 hari & Desember 2020 & harga sama dengan bulan Ialu \\
\hline 3 & 2021 & Agustus & 20 & Bener Meriah & Cabe merah & Keriting Segar & Faisal Tanjung & Desa Blang Paku & Via phone & 0823-6108-6303 & 17.000 & & 35 hari & Juli 2021 & 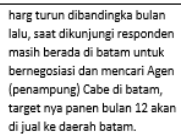 \\
\hline 4 & 2021 & Agustus & 20 & Bener Meriah & $\begin{array}{l}\text { Bawang } \\
\text { Merah }\end{array}$ & Ukuran Sedang & Tukimin & Dess Blang Paku & Ada & 0852-7717-8139 & 16.000 & & 4 Bulan & April 2021 & 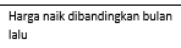 \\
\hline 5 & 2021 & Agustus & 20 & Bener Merizh & Cabe Merah & Keriting Segar & Tukimin & Desa Blang Paku & Ada & 0852-7717-8139 & 18.000 & & & & $\begin{array}{l}\text { saat ini belum tanam cabe, lahar } \\
\text { berisi tanaman nuang merah } \\
\text { rencana tanam cabe awal } \\
\text { September } 2021\end{array}$ \\
\hline 6 & 2021 & Agustus & 20 & Aceh Tengah & Cabe Merah & Keriting Segar & Suratno & Ketol Takengon & Via phone & 0852-7060-9498 & 18.000 & & & & $\begin{array}{l}\text { Harga turun nibandingkan hargas } \\
\text { bulan lalu, responden sedargn } \\
\text { dalam perjialnan ke batam }\end{array}$ \\
\hline 7 & 2021 & Agustus & 20 & Aceh Tengah & Cabe Rawit & Hijau Segar & Suratno & Ketol Takengon & \begin{tabular}{|l|} 
Via phone \\
\end{tabular} & \begin{tabular}{|l|}
$0852-7060-9498$ \\
\end{tabular} & 23.000 & & & & \begin{tabular}{|l|l} 
Harga naik dari bulan lalu \\
\end{tabular} \\
\hline
\end{tabular}

\begin{tabular}{|c|c|c|c|c|c|c|c|c|c|c|c|c|c|c|c|}
\hline No & Tahun & Bulan & $\mathrm{Tg}_{\mathrm{g}}$ & Kota//Kab & Komoditas & Kualitas & Produsen & Alamat & KTR Res & Hp & Harga & merek & $\begin{array}{c}\text { umur } \\
\text { tanaman/ternak }\end{array}$ & 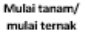 & Keterangan \\
\hline 8 & 2021 & Agustus & 20 & Aceh Tengesh & Cabe Merah & Kerriting Segar & Edy & Ketol Takergan & Via phone & $0821-6620-2208$ & 18.000 & & & & 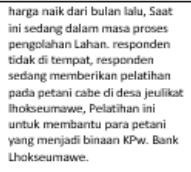 \\
\hline 9 & 2021 & Agustus & 20 & Aceh Tengash & Cabe Rawit & Hijisu Segar & Edy & Ketol Takengan & Via phone & \begin{tabular}{|l|l|}
$0821-6820-2208$ \\
\end{tabular} & 23.000 & & & & Harga naik dari bulan lasuu \\
\hline \multirow[b]{2}{*}{10} & \multirow[b]{2}{*}{2021} & \multirow[b]{2}{*}{ Agustus } & \multirow[b]{2}{*}{19} & \multirow[b]{2}{*}{ Bireuen } & \multirow[b]{2}{*}{ Dosing Sapi } & Kualitas 1 & \multirow[b]{2}{*}{ Iswandivrijal } & \multirow[b]{2}{*}{ suli } & \multirow[b]{2}{*}{ Ada } & \multirow[b]{2}{*}{$0813-6021-5885$} & 145.000 & & & & \multirow{2}{*}{ 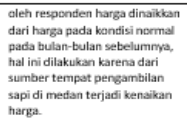 } \\
\hline & & & & & & Kualitas 2 & & & & & 1400000 & & & & \\
\hline 11 & 2021 & Agustus & 19 & Bireuten & Beras & kualitas medium 1 & M. Syakit & $\begin{array}{l}\text { Desa glarg Mee, Kutats } \\
\text { Blang }\end{array}$ & Ada & Oes2-6088-1535 & 9.870 & Majulara & & & $\begin{array}{l}\text { Harga naik ditbandingkan bulan } \\
\text { talu, harga gatath kering panen } \\
5.200 \text {, padi zak } 15 \text { g }_{1} 148.000 .\end{array}$ \\
\hline 12 & 2021 & Agustus & 19 & Aceh Utara & $\begin{array}{c}\text { Daging } \\
\text { Aysam Ras }\end{array}$ & Segar & Haffiuddin & Desa Pulo Fungkom & Ada & OBS2-6067-3136 & 14.000 & & & & $\begin{array}{l}\text { harga sama dengan bulan lalu, } \\
\text { harga pata swal azustus Rp. } \\
16.000\end{array}$ \\
\hline 13 & 2021 & Agustus & 19 & Aceh Utara & $\begin{array}{c}\text { Daging } \\
\text { Avram Ras }\end{array}$ & Segar & $\begin{array}{l}\text { Tgke. Har/Tgk. } \\
\text { MaNur }\end{array}$ & Desas Pinto Makmur & Ada & $008236363-2142$ & 14.000 & & 20 Hari & Agustus 2021 & harga sama dengan bulan lalu \\
\hline 14 & 2021 & Agustus & 19 & Aceh Utara & $\begin{array}{l}\text { Telur Ayam } \\
\text { Ras }\end{array}$ & Segar & $\begin{array}{l}\text { Tgk. Harr/gke } \\
\text { M.Nur }\end{array}$ & Dess Pinto Makmur & Ada & O823-6363-2142 & 20.550 & & & Januari 2020 & $\begin{array}{l}\text { Hargat turun ditbandingkan bulan } \\
\text { lalu. Harga } 1 \text { popan (30 butir Rp. } \\
39.000) \text {. }\end{array}$ \\
\hline 15 & 2021 & Agutus & 19 & Aceh Utara & Beras & $\begin{array}{l}\text { kualitas Premium } \\
\text { dan medium } 1\end{array}$ & $\begin{array}{c}\text { Amiruddin } \mathrm{K}_{p} \\
\text { Mandiri }\end{array}$ & Dess Tanjjang Messid & diwakili & 0813-174566881 & $\begin{array}{r}\text { Kualitas } \\
\text { premium Rp } \\
10,400 \\
\text { kualitas } \\
\text { medium } 1 \text { Rp } \\
10,000\end{array}$ & 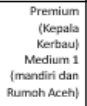 & & & harga sama dengan bulan lalu \\
\hline
\end{tabular}




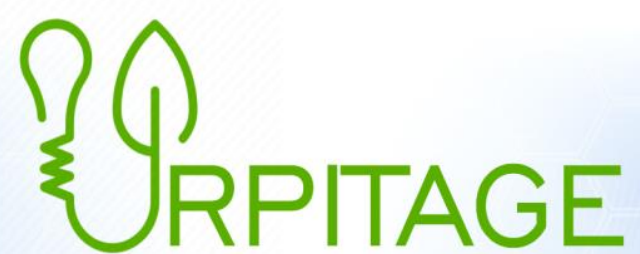

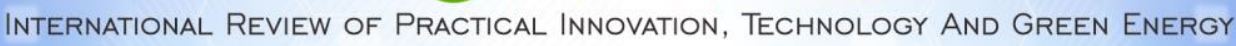

\begin{tabular}{|c|c|c|c|c|c|c|c|c|c|c|c|c|c|c|c|}
\hline No & Tahun & Butan & T:Aí & Kota///ab & Komoditas & Kualitas & Protusen & Alament & KTR Res & Hp & Hesa & merek & 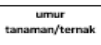 & $\begin{array}{l}\text { Molui tanamar } \\
\text { mutai termak }\end{array}$ & Kenteransan \\
\hline 16 & 2021 & 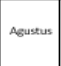 & 19 & Aach Utara & $\begin{array}{l}\text { Dagign } \\
\text { Arem Ras }\end{array}$ & Segar & Nurtain Restan & cot trueng & 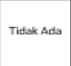 & 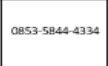 & & & & & 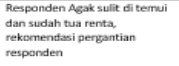 \\
\hline${ }^{17}$ & 2021 & Agurusus & ${ }^{19}$ & unatecumane & Desing Sapi & $\begin{array}{l}\text { Kualtas } 1 \\
\text { Kualtas } 2 \\
\end{array}$ & 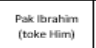 & 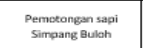 & Via phone & | $0052-6229.9653$ & $\begin{array}{l}145.000,00 \\
1500000\end{array}$ & & & & 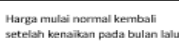 \\
\hline 18 & 2021 & 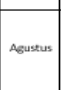 & 19 & Bireuen & Beras & kualitas medium 1 & Insen & Gungeok ganda pura & diwakali & 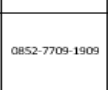 & 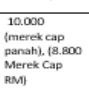 & 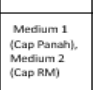 & & & Harga somo dengan bulan Lalu \\
\hline 19 & 2021 & 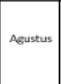 & 19 & Lhokeceumaume & Cate Meran & Xeriting Segar & test & 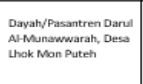 & Ada & 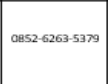 & 20.000 & & & & 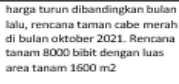 \\
\hline 20 & 2021 & Aguress & \begin{tabular}{c|c}
19 \\
19
\end{tabular} & Aceh Timur & Cobe Ravit & hisiow segar & Agers & Pusong Sematang & Via phone & OA22-74a3-0038 & 23.000 & & & & 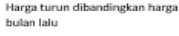 \\
\hline${ }^{21}$ & 2021 & Agurus: & ${ }_{19}^{19}$ & Aceh Utara & 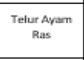 & Segar & Dani & Alang falleun & 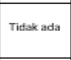 & 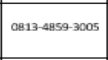 & & & & & 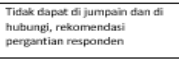 \\
\hline 22 & 2021 & Agurus & 20 & Bener Meriah & cosati Rmit & Hijizu S Segar & Mathara Putra & Getampang With & \begin{tabular}{|l|l|l|l} 
va phone & \\
\end{tabular} & | $0123-727292-2860 \mid$ & 23.000 & & 3,5 butan & Mei 2021 & 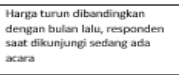 \\
\hline${ }^{23}$ & 2021 & Asumes: & 20 & Bener Meriath & $\begin{array}{l}\text { Boweng } \\
\text { Merath }\end{array}$ & Ukuran Sedang & sugianta & Suka makmur & Via phone & Oez2-7539-7078 & 12.000 & & 34 hari & Jus 2021 & 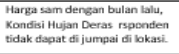 \\
\hline 24 & 2021 & \begin{tabular}{|l|l|l|l|l} 
Asurus & \\
\end{tabular} & 20 & Aceh Tengesh & 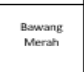 & Ukuran Setang & Batin & Gunung Bashenen & \begin{tabular}{|l|l|} 
Vis phone & |
\end{tabular} & \begin{tabular}{|c|c|}
$0622.7539-7078$ \\
\end{tabular} & & & 95 thai & April 2021 & 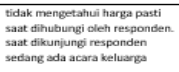 \\
\hline 25 & 2021 & Agutus & 19 & Bireuen & Beras & Kualitas Medium 1 & Jutheman & Kuneng Panjoe & Va phone & |0852-7764-4553 & 9.875 & Rajajwati & & & 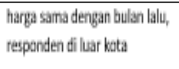 \\
\hline
\end{tabular}

Figure 1. Documentation of the Aceh Province Leading Commodities Survey as an Effort for Academics to Participate in Developing the Nation
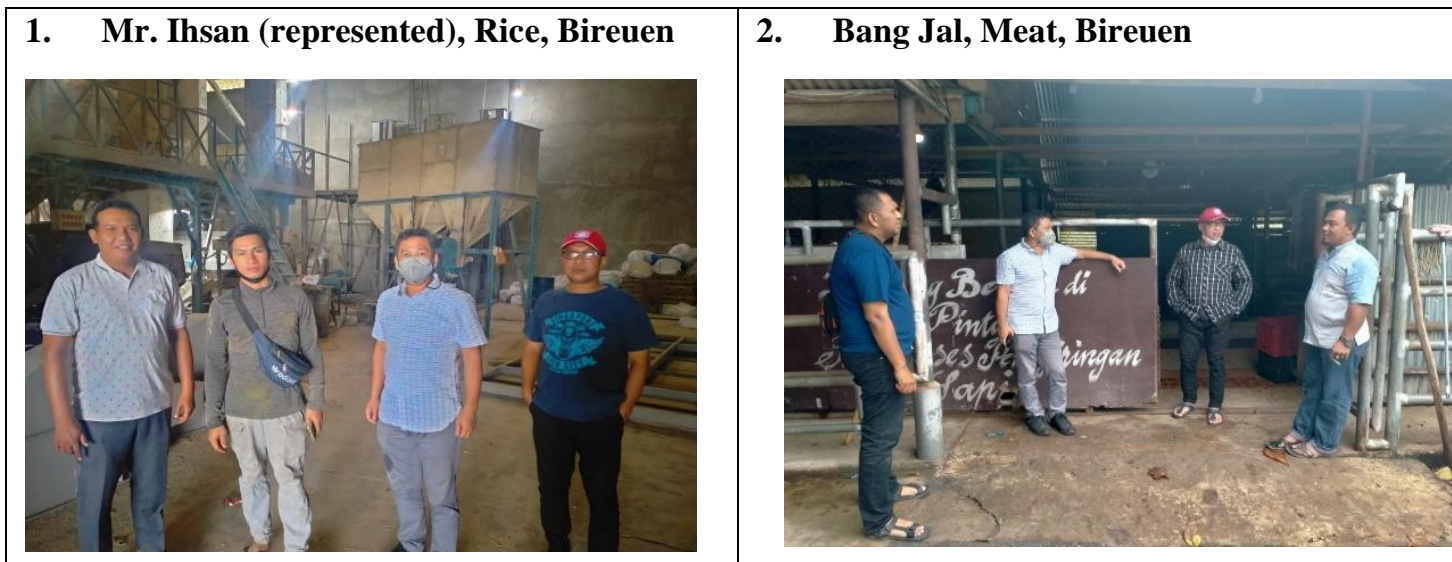

\section{Hafifuddin, Chicken, North Aceh}

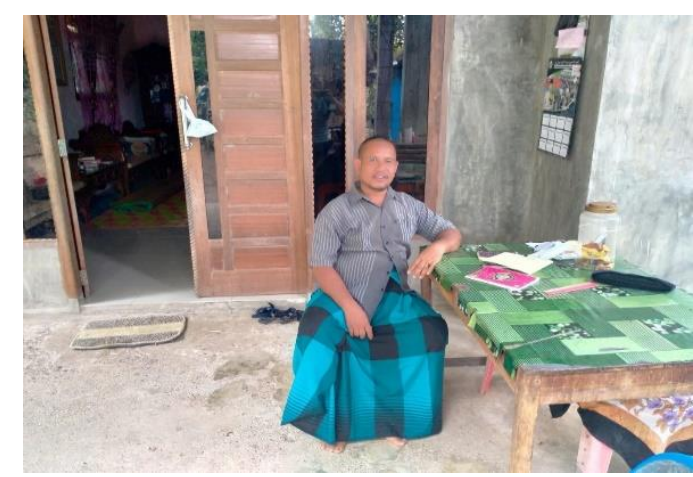

\section{Ilyas, Red \& Rawit Chili, East Aceh}

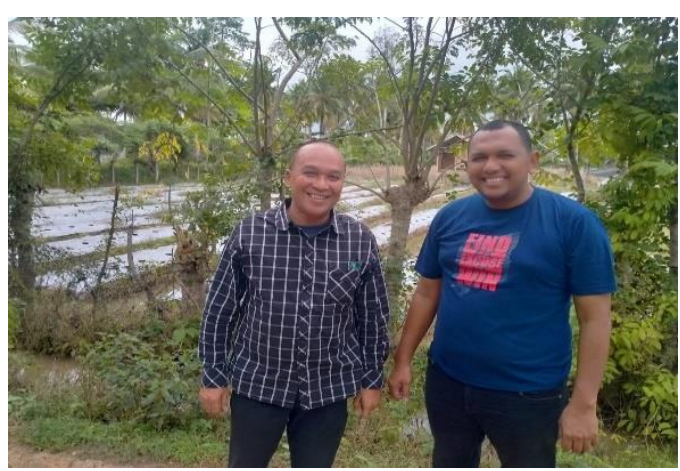




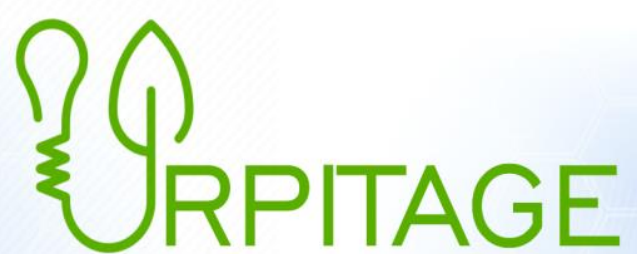

INTERnATIONAL REVIEW of PRACTICAL INNOVATION, TECHNOLOGY AND GREEN ENERGY

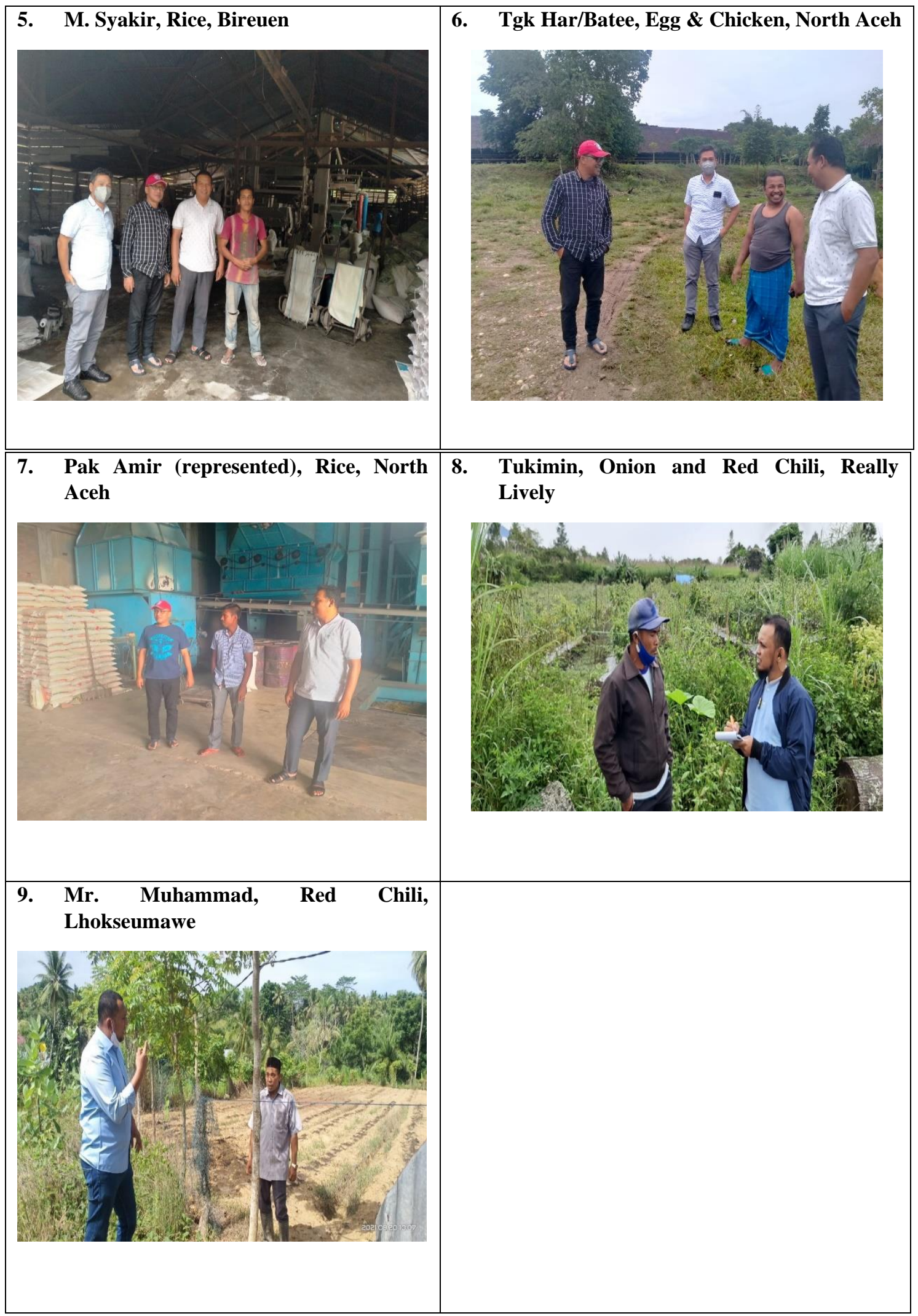




\section{REFERENCE}

Ernan, R. Juli \pm Agustus, 2009. Penataan Ruang Kawasan Perdesaan dan Agropolitan Sebagai Strategi Pembangunan Perdesaan. Tata Ruang: 28-33.

Ernan, R., Sunsun, S. dan Dyah R. P. 2011. Perencanaan dan Pengembangan Wilayah. Jakarta. Crestpent Press dan Yayasan Pustaka Obor Indonesia, Anggota IKAPI DKI Jakarta.

Jefri Leo, 2014. Penentuan Komoditas Unggulan Pertanian dengan Metode Analitycal Hierarchy Process (AHP). Jurnal Saintia Matematika, Volume 02, Nomor 03 (2014), ISSN : 2337-9197.

Perpustakaan Nasional, Republik Indonesia, 2014. Peraturan Perundang-undangan. Cetakan Pertama. CV. Eka Jaya, Jakarta 13410. 